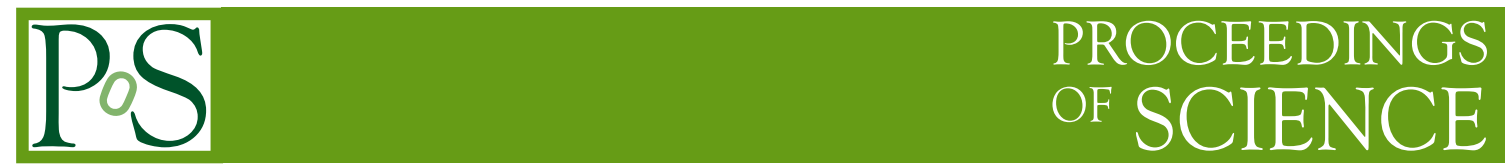

\title{
Search for Branons at LEP
}

\section{Salvatore Mele*}

INFN, Sezione di Napoli, I-80125, Napoli, Italy

E-mail: Salvatore.Mele@cern.ch

Branons are natural dark-matter candidates predicted by theories with extra dimensions. They correspond to the fluctuation of a three-dimensional brane along the extra dimensions and could be the first manifestation of this new physics in scenarios where gravitons are not accessible at high-energy colliders. Two searches performed with the L3 detector at LEP for branon production in the single- $\mathrm{Z}$ and single-photon channels are discussed. Their results allow to derive the most stringent limits on the possible existence of branons.

International Europhysics Conference on High Energy Physics

July 21st - 27th 2005

Lisboa, Portugal

${ }^{*}$ Speaker. 


\section{Introduction}

The scale of gravitational phenomena, $m_{P l} \approx 10^{19} \mathrm{GeV}$, is immensely larger than the scale of the electroweak interactions, $m_{e w} \approx 10^{2} \mathrm{GeV}$, leading to the so-called hierarchy problem. Some recent theoretical ideas introduce mechanisms to reduce the scale of gravity to a new scale, $M_{F}$, of the order of $m_{e w}$ [1]. This natural solution of the hierarchy problem postulates the existence of extra space dimensions. These could manifest themselves as deviations from the Newton law in short-range tests of gravity or in new phenomena at high-energy colliders mediated by gravitons. No such deviations have been observed so far.

In a different scenario, the presence of a three-dimensional brane as an additional physical body in a theory with extra dimensions implies additional degrees of freedom associated to its fluctuation along the extra dimensions [2]. These degrees of freedom manifest as branons: new scalar particles, $\tilde{\pi}$, described by an effective theory with couplings of the same order as the brane tension, $f$. Branons are natural dark-matter candidates [3].

To some extent, searches for gravitons and branons are complementary [4]. For $f \gg M_{F}$, the first evidence for extra dimensions would be the discovery of gravitons. For $f \ll M_{F}$, gravitons might not be accessible at high-energy colliders and branons would provide the first signal of extra dimensions.
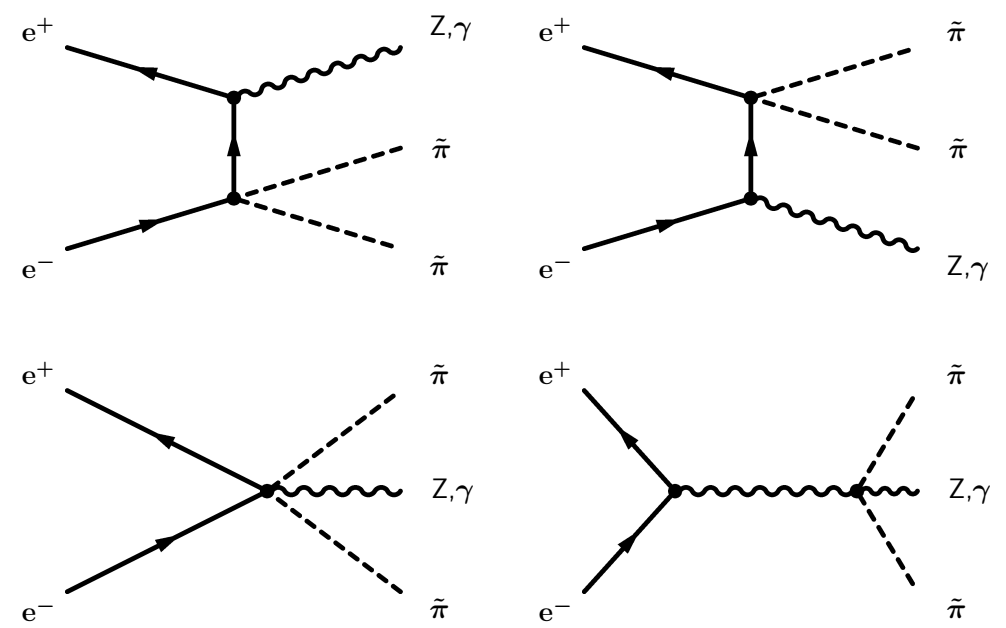

Figure 1: Feynman diagrams describing branon production at LEP through the $\mathrm{e}^{+} \mathrm{e}^{-} \rightarrow \tilde{\pi} \tilde{\pi} \mathrm{Z}$ and $\mathrm{e}^{+} \mathrm{e}^{-} \rightarrow$ $\tilde{\pi} \tilde{\pi} \gamma$ processes.

\section{Branon manifestations at LEP}

Branons couple to Standard Model particles in pairs and could be produced in $\mathrm{e}^{+} \mathrm{e}^{-}$collisions at LEP through the Feynman diagrams shown in Figure 1. They do not interact in the detector giving a signature of missing energy and momentum associated with either a single photon or a single Z-boson.

The L3 Collaboration searched for branon production at LEP in a data sample of about $0.6 \mathrm{fb}^{-1}$ collected at centre-of-mass energies $\sqrt{s}=189-209 \mathrm{GeV}$ [5], with an average value $\langle\sqrt{s}>=$ 
$198 \mathrm{GeV}$. Events with a single Z-boson decaying into hadrons and missing energy and momentum are selected to tag the $\mathrm{e}^{+} \mathrm{e}^{-} \rightarrow \tilde{\pi} \tilde{\pi} \mathrm{Z} \rightarrow \tilde{\pi} \tilde{\pi} \mathrm{q} \overline{\mathrm{q}}$ process, while events with a single photon and missing energy and momentum are used to search for the $\mathrm{e}^{+} \mathrm{e}^{-} \rightarrow \tilde{\pi} \tilde{\pi} \gamma$ process. The methods and results of these analyses are discussed in the following sections.

\section{Search in the single- $Z$ channel}

The analysis selects unbalanced hadronic events with a visible mass compatible with that of a $\mathrm{Z}$ boson. The main background sources are: $\mathrm{e}^{+} \mathrm{e}^{-} \rightarrow \mathrm{q} \overline{\mathrm{q}} \gamma$ events with an undetected low-angle high-energy photon; pair-production of $\mathrm{W}$ bosons with a semileptonic final state where a lepton escapes detection; $\mathrm{Z}$ boson pair-production followed by the decay of a $\mathrm{Z}$ boson into hadrons and the other into neutrinos; single W-boson production.

A requirement on the missing momentum vector to point in the detector strongly suppresses background from the $\mathrm{e}^{+} \mathrm{e}^{-} \rightarrow \mathrm{q} \overline{\mathrm{q}} \gamma$ process. Other backgrounds are reduced by selection criteria on event-shape and jet-shape variables. A total of 455 events is observed in data, while 470 events are predicted by Standard Model processes. A branon signal with $M=0 \mathrm{GeV}$ and $f=40 \mathrm{GeV}$ would be selected with a 55\% efficiency and contribute with 123 events to this sample.

The two variables most sensitive to a possible branon signal are the reduced energy of the reconstructed $\mathrm{Z}$ boson, $x_{\mathrm{Z}}=E_{\mathrm{Z}} / \sqrt{s}$, and the cosine of its polar angle, $\theta_{\mathrm{Z}}$. The distribution of these variables for data and Standard Model Monte Carlo are shown in Figures 2a and 2b, respectively, together with the predictions in presence of a branon signal. No excess is observed.
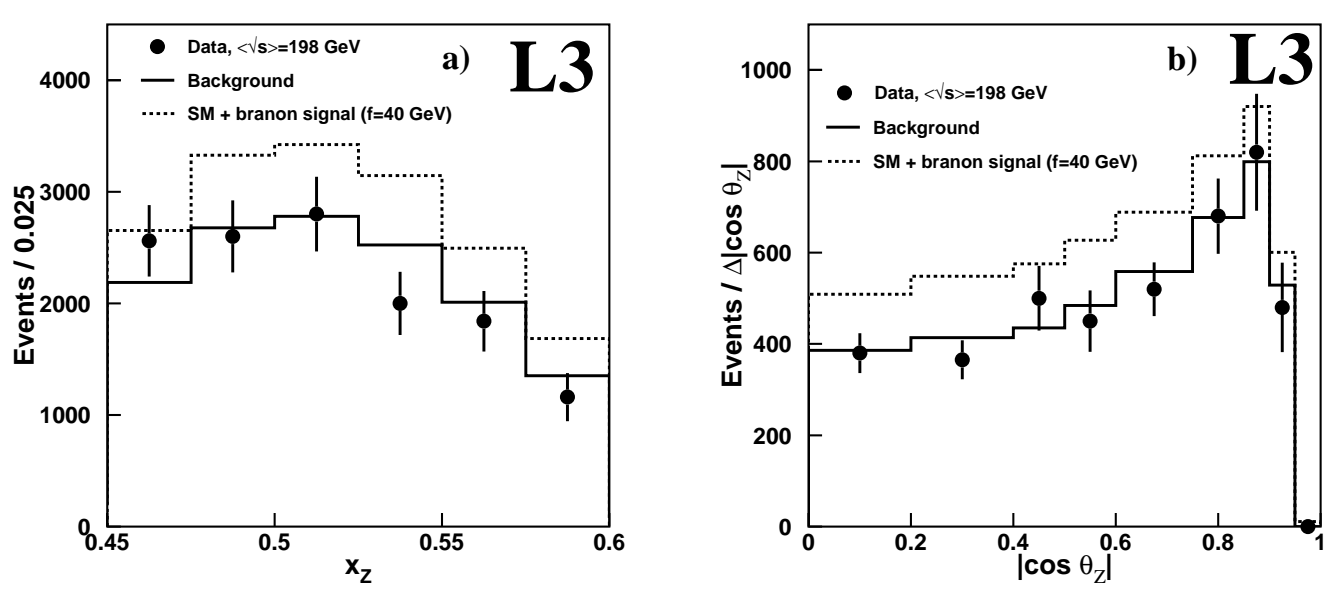

Figure 2: Distributions of a) the reduced energy and b) the cosine of the polar angle of single Z-boson candidates. Data and Standard Model Monte Carlo are shown together with the expected signal for massless branons with $f=40 \mathrm{GeV}$

\section{Search in the single-photon channel}

The L3 Collaboration published a high-precision study of events with a single photon and 
missing energy [6] in two different energy regions, according to the value of the transverse momentum of the photon, $p_{t}$, relative to the beam energy, $E_{\text {beam }}$. A high- $p_{t}$ study covers the range $0.04<p_{t} / E_{\text {beam }}<0.60$ in the barrel and endcap regions of the high-precision BGO electromagnetic calorimeter, $\left|\cos \theta_{\gamma}\right|<0.73$ and $0.8<\left|\cos \theta_{\gamma}\right|<0.97$, respectively. A low- $p_{t}$ selection allows the study of events with $0.016<p_{t} / E_{\text {beam }}<0.04$ in the barrel region.

The high- $p_{t}$ selection observes 838 events in data while 811 events are expected from Standard Model processes, corresponding to a $\mathrm{e}^{+} \mathrm{e}^{-} \rightarrow v \bar{v} \gamma$ signal with a purity of $99 \%$ and an efficiency of $80 \%$. Figure 3a compares the observed cross section of the $\mathrm{e}^{+} \mathrm{e}^{-} \rightarrow v \bar{v} \gamma$ process as a function of $x_{\gamma}=E_{\gamma} / E_{\text {beam }}$ with the prediction of the Standard Model and the possible excess of a branon signal with $M=0 \mathrm{GeV}$ and $f=150 \mathrm{GeV}$. The data are in good agreement with the Standard Model prediction.

The low- $p_{t}$ selection observes 543 events in data whereas 554 events are expected from Standard Model processes, three quarters are from the $\mathrm{e}^{+} \mathrm{e}^{-} \rightarrow \mathrm{e}^{+} \mathrm{e}^{-} \gamma$ process and the remaining from the $\mathrm{e}^{+} \mathrm{e}^{-} \rightarrow v \bar{v} \gamma$ process. Figure $3 \mathrm{~b}$ compares the observed and expected distributions of $x_{\gamma}$. A good agreement is observed. The excess expected in presence of a possible branon signal with $M=0 \mathrm{GeV}$ and $f=150 \mathrm{GeV}$ is also shown.
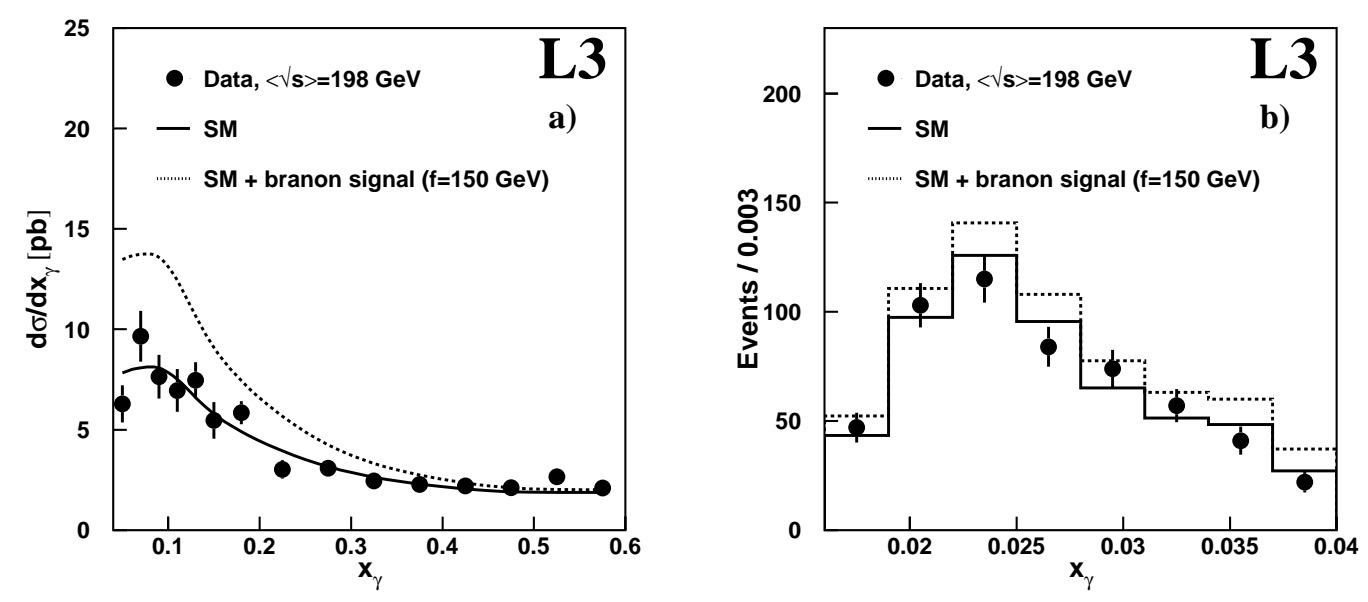

Figure 3: Observed and expected a) cross sections of the $\mathrm{e}^{+} \mathrm{e}^{-} \rightarrow v \bar{v} \gamma$ process from the high- $p_{t}$ singlephoton selection and $\mathrm{b}$ ) distributions of the reduced photon energy observed by the low- $p_{t}$ selection. The expected signals for massless branons with $f=150 \mathrm{GeV}$ is also shown

\section{Results}

No evidence for branon production was found either in the $\mathrm{e}^{+} \mathrm{e}^{-} \rightarrow \tilde{\pi} \tilde{\pi} \mathrm{Z}$ or in the $\mathrm{e}^{+} \mathrm{e}^{-} \rightarrow \tilde{\pi} \tilde{\pi} \gamma$ channel. Two-dimensional distributions of $x_{\mathrm{Z}} v s \cdot \cos \theta_{\mathrm{Z}}$ for the single-Z channel and of $x_{\gamma} v s \cdot \cos \theta_{\gamma}$ for the single-photon channel are compared with the double-differential cross section for branon production [7] and 95\% confidence level limits are extracted as a function of $M$ and $f$, as shown in Figure 4. 
The analysis of the $\mathrm{e}^{+} \mathrm{e}^{-} \rightarrow \tilde{\pi} \tilde{\pi} \mathrm{Z}$ channel results in $f>47 \mathrm{GeV}$ for massless branons. There is no sensitivity for branon masses near and beyond the kinematic limit $M \gtrsim\left(\sqrt{s}-m_{\mathrm{Z}}\right) / 2$ and no bounds on $f$ can be derived for $M>54 \mathrm{GeV}$.

The $\mathrm{e}^{+} \mathrm{e}^{-} \rightarrow \tilde{\pi} \tilde{\pi} \gamma$ channel has a larger sensitivity owing to the larger photon-electron coupling and a larger phase space available for a final state photon instead of a massive $\mathrm{Z}$ boson. For $M=0$, a limit $f>180 \mathrm{GeV}$ is established. For very elastic branes, $f \rightarrow 0$, a lower mass limit is obtained as $M>103 \mathrm{GeV}$.

These bounds are the most stringent to date on the possible existence of branons. They complement and improve those deduced from astrophysical observations [3].

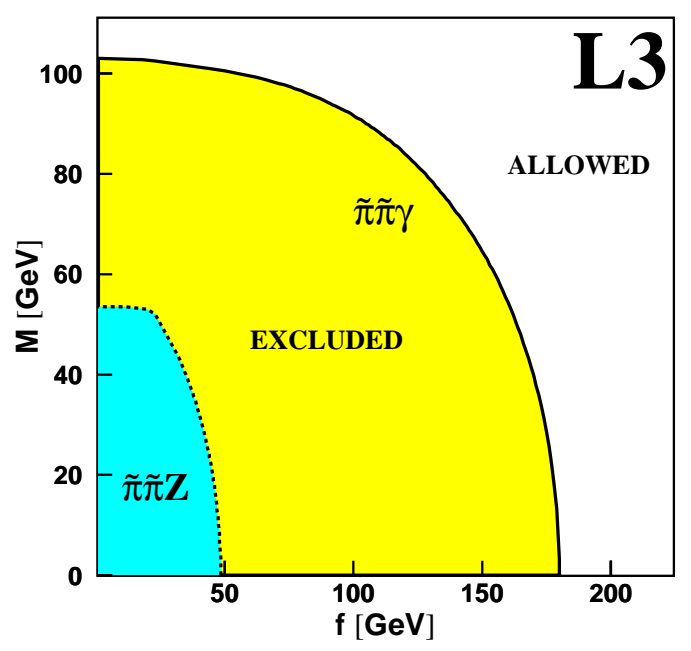

Figure 4: Exclusion regions in the plane of the branon mass, $M$, and brane tension, $f$, derived by the $\mathrm{e}^{+} \mathrm{e}^{-} \rightarrow \tilde{\pi} \tilde{\pi} \mathrm{Z}$ and $\mathrm{e}^{+} \mathrm{e}^{-} \rightarrow \tilde{\pi} \tilde{\pi} \gamma$ searches.

\section{References}

[1] N. Arkani-Hamed, S. Dimopoulos and G. Dvali, Phys. Lett. B 429 (1998) 263;

I. Antoniadis et al., Phys. Lett. B 436 (1998) 257;

L. Randall and R. Sundrum, Phys. Rev. Lett. 83 (1999) 3370.

[2] R. Sundrum, Phys. Rev. D59 (1999) 085009;

A. Dobado and A.L. Maroto, Nucl. Phys. B 592 (2001) 203.

[3] J.A.R. Cembranos, A. Dobado and A.L. Maroto, Phys. Rev. Lett. 90 (2003) 241301;

J.A.R. Cembranos, A. Dobado and A.L. Maroto, Phys. Rev. D68 (2003) 103505.

[4] M. Bando et al., Phys. Rev. Lett. 83 (1999) 3601.

[5] L3 Collab., P. Achard et al., Phys. Lett. B 597 (2004) 145.

[6] L3 Collab., P. Achard et al., Phys. Lett. B 584 (2004) 16.

[7] J. Alcaraz et al., Phys. Rev. D67 (2003) 075010. 